

\title{
SISTEMAS DE INFORMAÇÃO NA ÁREA DA SAÚDE: A INFORMAÇÃO CLÍNICA COMO INSTRUMENTO DE TRABALHO PARA OS PROFISSIONAIS DE SAÚDE
}

Chennyfer Dobbins Paes da Rosa

Doutora em Saúde Coletiva, Mestre em Economia da Saúde pela Universidade Federal de São Paulo (UNIFESP). Docente do Programa de Mestrado Profissional em Gestão em Sistemas de Saúde - Universidade Nove de Julho (UNINOVE).E-mail: chennyferr@yahoo.com.br.

\section{Denise Mathias}

Mestranda do Programa de Mestrado Profissional em Gestão em Sistemas de Saúde - Universidade Nove de Julho (UNINOVE).

\section{Ricardo Leonardo Rovai}

Doutor em Engenharia de Produção pelo Departamento de Engenharia de Produção da Escola Politécnica da Universidade de S.Paulo. Mestre em Administração pela FEA - USP. Professor do Mestrado Profissional em Gerenciamento de Projetos - Universidade Nove de Julho (UNINOVE). Pesquisador do PGT-USP.

Resumo: A garantia de uma assistência de qualidade apresenta-se como um dos principais objetivos dos Sistemas de Informação clínicos, auxiliando nas tomadas de decisão entre os vários profissionais envolvidos na assistência ao cliente. A continuidade dos cuidados é um dos aspetos essenciais nos processos de avaliação da qualidade dos serviços de saúde, o que afirma a importância de informações clínicas neste contexto. O objetivo deste trabalho é descrever o significado da informação clínica, como instrumento de trabalho dos profissionais de saúde, para melhor Prática Baseada em Evidência (PBE). Trata-se de um estudo teórico, cuja base de coleta de dados foi a base Scielo. Nos resultados de busca dos artigos indexados nesta base foram de 115 artigos, sendo 12 incluídos para descrever a informação clínica como instrumento de trabalho. Entendeu-se que é necessária uma boa fonte de dados e o profissional estar atento às informações de qualidade. Conclui-se que a informação clínica é um instrumento essencial para uma adequada conduta assistencial, porém é necessário organizar as informações de maneira que sejam eficazes para a tomada de decisão.

Palavras-chaves: Sistema de Informação em Saúde. Sistemas de Informação. Sistemas de Informação Hospitalar.

\section{POLÊM!CA | Revista Eletronica da Ueji}




\title{
INFORMATION SYSTEMS IN HEALTH: CLINICAL INFORMATION FOR HEALTH PROFESSIONALS
}

\begin{abstract}
Ensuring the quality of care is presented as a major goal of clinical information systems, assisting in decision-making between the various professionals involved in customer service. Continuity of care is one of essesnciais aspects in the procedures for assessing the quality of health services, which affirms the importance of clinical information in this context. The objective of this study is to describe the meaning of the clinical information as a working tool for health professionals to better Evidence Based Practice (EBP). This is a theoretical study, the data collection was based Scielo. In search results of articles indexed on this basis were 115 articles of which 12 included to describe the clinical information as a working tool. It was understood that a good source of data is required and the professional to be aware of the quality of information. We conclude that clinical information is an essential tool for proper conduct but care is necessary to organize the information so that is effective for the decision making.
\end{abstract}

Keywords: Health Information Systems. Information Systems. Hospital Information Systems.

\section{Introdução}

As organizações para que sobrevivam no mercado, precisam gerenciar suas informações de maneira que subsidiem as tomadas de decisão eficazes. Sabe-se que o gerenciamento das informações é usual nas instituições, tanto que pessoas de diferentes formações (administradores, engenheiros, economistas, enfermeiros, médicos, dentre outras) são contratadas para essa finalidade.

Os Sistemas de Informação (SI) são sistemas ou processos que transformam dados em informações que auxiliam nas tomadas de decisão, sejam assistenciais ou gerenciais, de forma eficiente, objetiva e rápida, por isso são cada vez mais implementados. Para que os resultados sejam bem-sucedidos é necessária a interação colaborativa entre pessoas, tecnologias e procedimentos (RODRIGUES, 2000).

Os SI são cada vez mais necessários nas organizações e essa realidade também está inserida nos serviços de saúde. Os SI constituem de um recurso estratégico, no que diz respeito às ações tanto nas tomadas de decisão clínicas por parte dos profissionais de saúde, como governamentais pensando nas políticas de saúde pública (MOTA, PEREIRA, SOUSA, 2014).

No contexto do setor da saúde os SI devem ser vinculados à garantia de qualidade, pois devem agregar uma informação útil às diversas funções dos profissionais de saúde e aos diferentes níveis de decisão da instituição (MOTA, PEREIRA, SOUSA, 2014).

Os SI devem ser estruturas sólidas, capazes de garantir processos eficientes com o devido armazenamento, processamento, organização e gestão dos dados que resultam dos processos assistenciais (MOTA, PEREIRA, SOUSA, 2014). 
É fundamental que os sistemas de informação sejam simples, com acesso fácil e rápido aos dados, que estes sejam adequadamente armazenados e que processem informações relevantes, facilitando a utilização na tomada de decisão. (MOTA, PEREIRA, SOUSA, 2014).

É neste sentido que as estratégias de utilizar ferramentas que auxiliem no trabalho dos profissionais de saúde devem ser essenciais, buscando a melhoria da qualidade dos cuidados prestados ao paciente (MOTA, PEREIRA, SOUSA, 2014).

Na área da saúde, a informação é fonte essencial para melhor prática assistencial, entender os SI e utilizá-los na rotina, faz com que o profissional de saúde consiga agilizar o atendimento ao paciente (RODRIGUES, 2000).

A implementação de um SI permite melhorar a prática clínica, adequar os cuidados de saúde e aumentar a eficiência e efetividade das organizações de saúde.

Os SI são locais de armazenamento de dados dos pacientes, em suporte eletrônico, que permitem o compartilhamento de informações entre diversos colaboradores, devidamente autorizados (MOTA, PEREIRA, SOUSA, 2014).

Refletindo sobre a utilização do SI e as ferramentas que podemos utilizar na prática clínica, uma delas é a Informação Clínica (IC).

A Informação Clínica (IC) é qualquer informação produzida ou utilizada por profissionais da área de saúde (médico, enfermeiro, fisioterapeuta, nutricionista, terapeuta ocupacional, fonoaudiólogo, dentre outros) em busca de diagnosticar, avaliar, tratar ou recuperar as condições de saúde dos indivíduos que buscam assistência. A informação clínica precisa ser clara, objetiva, efetiva, ter qualidade e segurança, pois a mesma pode agravar as condições de saúde do paciente se não fidedigna (GALVÃO, 2012).

O presente trabalho tem por intenção descrever o significado da informação clínica como instrumento de trabalho dos profissionais de saúde para melhor Prática Baseada em Evidência (PBE).

\section{Referencial Teórico - Prática Baseada em Evidência (PBE)}

Prática Baseada em Evidência (PBE) é um conceito utilizado para explicitar o uso crítico, consciencioso e direcionado sobre a melhor evidência científica disponível sobre determinado assunto, auxiliando para a tomada de decisão assistencial/ clínica (SACKETT, 1998).

\section{POLÊM!CA | Revista Eletronica da Ueji}


É uma metodologia que auxilia os profissionais da área de saúde a tomarem suas decisões clínicas dentro do campo científico.

O termo primeiramente foi utilizado na Medicina, como Medicina Baseada em Evidência, e, posteriormente, utilizado pelas demais áreas da saúde como Prática Baseada em Evidência (CENTRE, 2015).

A Prática Baseada em Evidências (PBE) surgiu de volta em 1947, quando o Sr. James Lind, da Inglaterra, lutou contra o escorbuto durante viagens longas. Ele decidiu introduzir as experiências bem descritas em prática. Depois de tentar seis abordagens diferentes para tratar o escorbuto, ele observou que limão e suco de laranja produziam cura. Somente 40 anos depois, quando o uso de suco de limão e laranja foi introduzido na dieta do grupo, em 1835, o Sr. Pierre fez a primeira verificação de um tratamento com análise estatística. Ele comparou grupos de indivíduos com pneumonia. Um grupo foi submetido a tratamento padrão, outro usando sanguessugas e um terceiro grupo considerado como grupo controle, sem sanguessugas. Ele constatou que os pacientes em ambos os grupos, controle e intervenção, morreram. No entanto, o grupo controle tive menor mortalidade (CENTRE, 2015).

No Brasil, os primeiros relatórios sobre o uso de diretrizes surgiram em 2000 pela ANS (Agência de Saúde Suplementar), o objetivo principal foi de ajudar a administração de seguros privados de saúde a desenvolver políticas de saúde e boas práticas clínicas, além de desenvolverem protocolos para autorização de procedimentos assistenciais realizados nos serviços de saúde a seus usuários. Essas diretrizes incluíram basicamente procedimentos e materiais de alto custo; houve falta de sistematização e regulamentação por ser algo novo, pouco utilizado na época (ASSOCIAÇÃO MEDICA BRASILEIRA (AMB), CONSELHO FEDERAL DE MEDICINA, AGÊNCIA NACIONAL DE SAÚDE SUPLEMENTAR, 2009).

Entre os vários objetivos para usar diretrizes baseadas em evidências, destacamos a promoção da qualidade dos cuidados de saúde, estimulando a pesquisa clínica e médica, avaliando os benefícios de vários tipos de abordagens alternativas, apoiando as decisões em matéria de política de saúde e prestação de normas de boa prática com opções baseadas em evidências, que permitam decisões clínicas adequadas (ASSOCIAÇÃO MEDICA BRASILEIRA, 2009).

A implantação das diretrizes utilizadas para a prática clínica diária é um desafio. Cabe aos gestores e administradores de serviços de saúde promover a divulgação da relevância da 
Prática Baseada em Evidência, conscientizando os profissionais de saúde sobre a necessidade da sua aplicação na rotina diária de trabalho (ASSOCIAÇÃO MEDICA BRASILEIRA (AMB), CONSELHO FEDERAL DE MEDICINA, AGÊNCIA NACIONAL DE SAÚDE SUPLEMENTAR, 2009).

Distribuir materiais educativos para os profissionais de saúde; distribuição de recomendações publicadas ou impressas para atendimento clínico, incluindo orientações para a prática clínica, materiais audiovisuais e publicações eletrônicas; encontros educacionais com os prestadores de cuidados de saúde, como conferências, palestras, oficinas ou estágios bem como os processos de consenso locais, são alguns dos meios importantes de divulgação da informação clínica (SACKETT, 1998).

A informação clínica, para ser usada como ferramenta de trabalho dos profissionais de saúde, deve vir com a aplicabilidade prática; ela deve apoiar as discussões com os pacientes, ou o envolvimento do paciente na tomada de decisão; informar especificações técnicas e impacto previsto, além de trazer a possibilidade de análises de estratégias de implementação, identificando as barreiras de uso e seleção, otimizando os custos, aumentando a eficiência do atendimento ao paciente, aumentando a eficácia, contribuindo para a melhoria dos cuidados de saúde (RODRIGUES, 2000).

\section{Fontes de Informação Clínica}

Existem algumas fontes de informação clínica, dentre elas o Prontuário do Paciente, este pode ser eletrônico ou impresso em papel.

O prontuário é um documento que pela natureza de seu conteúdo sofre restrição de acesso (GALVÃO E RICARTE, 2012). As informações relacionadas à saúde de um paciente devem ser sigilosas e podem ser utilizadas pela equipe multiprofissional durante a relação profissional (GALVÃO E RICARTE, 2012).

O Prontuário Eletrônico do Paciente (PEP) é o conjunto de informações sobre o estado e cuidado de saúde, ao longo da vida de um paciente, armazenado eletronicamente e pode incluir além das informações resultantes da atenção ao paciente, outras funções não disponíveis no prontuário em papel: alertas e lembretes, módulo de crítica da prescrição médica, ligações com bases de conhecimentos para apoio à decisão, incorporação de 
protocolos clínicos, interfaces adaptadas pelo usuário, integração com os laboratórios e farmácia, permitindo a integração de dados de pacientes (OLIVEIRA, 2011).

O objetivo do prontuário do paciente é assegurar serviços de saúde integrados de modo contínuo, eficiente e com qualidade, juntamente com informação retrospectiva, corrente e prospectiva. (GALVAO, RICARTE, 2012).

Existe o prontuário da família que é utilizado no Programa de Saúde da Família (PSF), este registro busca reunir informações clínicas de todos os membros de um núcleo familiar. Espera-se neste contexto que no futuro possamos ter por meio dos recursos tecnológicos um prontuário da comunidade que possa trazer a síntese de todas as condições de saúde referentes a um território (MUELLER, 2000).

O prontuário virtual do paciente também é outra expectativa de fonte de dados, pois poderá integrar todas as informações clínicas de um paciente, independente do local geográfico ou contexto clínico no qual ele tenha sido atendido (atenção primária, secundária ou terciária), a fim de se ter uma compreensão integral da condição de saúde do paciente (GALVAO, RICARTE, 2012)

Outra fonte de informação clínica aplicável ao contexto assistencial, muito utilizada pelos profissionais de saúde são as Bases de dados Especializadas. Essas são bibliotecas online que armazenam de forma sistemática artigos científicos, livros e teses. Trazem artigos científicos especializados em questões clínicas que podem contribuir para a busca da melhor Prática Baseada em Evidência (MUELLER, 2000).

Essas bases têm metodologias específicas para coleta da melhor informação, possibilitando uma triagem de evidências científicas que tenham um potencial efetivo para serem aplicadas na prática da assistência a um paciente ou a uma coletividade.

Existem inúmeras bases de dados, quando fazemos revisões sistemáticas, é necessário que a análise seja feita em todas elas, porém quando é para o desenvolvimento de protocolos, guidelines ou para si mesmo, tentando identificar o melhor nível de evidência, cabe ao pesquisador definir uma ou mais bases de acordo com o tempo disponível para a pesquisa.

Dependendo do assunto, a busca resultará em um número grande de artigos encontrados, portanto, a estratégia de busca auxiliará a atingir o objetivo proposto, sem perder a qualidade dos resultados, no menor tempo possível.

Dentre as bases de dados mais utilizadas, podemos destacar:

\section{POLÊM!CA | Revista Eletronica da Uej}


Textuais, nas quais os conteúdos são textos completos - exemplo, SciELO (Scientific Eletronic Library Online) (http://www.scielo.org) que é uma base de dados que inclui os principais periódicos brasileiros; nas quais o conteúdo apresenta referências de documentos (artigos de revistas, livros e teses) com ou sem resumos.

BestBETs tem o foco nos problemas clínicos relacionados com a medicina de emergência. Cada tópico apresentado na base de dados responde a uma questão clínica (BESTBETS, 2015).

Clinical Evidence descreve a melhor evidência disponível a partir de revisões sistemáticas, estudos clínicos randomizados e estudos observacionais. Informa quando não há nenhuma evidência disponível sobre o assunto. Esta base de dados também se propõe a apresentar aos profissionais de saúde e pacientes os motivos/os porquês de uma evidência ser adequada ou não a um dado contexto. (CLINICAL, 2015).

Cochrane Systematic Reviews congrega revisões sistemáticas de pesquisa essenciais para a saúde humana e para o estabelecimento de políticas de saúde. Inclui estudos que investigam os efeitos das intervenções de prevenção, promoção terapêutica e reabilitação, bem como acurácia de testes diagnósticos para uma determinada condição de saúde, para um determinado perfil ou grupo de pacientes, ou para um determinado contexto de tratamento de saúde (contexto de internação ou de tratamento ambulatorial) (COCHRANE, 2015).

DynaMed é uma ferramenta de referência clínica criada por médicos para médicos e outros profissionais de saúde para o uso no ponto da assistência ao paciente. A base de dados busca fornecer conteúdo atualizado e os recursos com relevância, validade e conveniência para responder a maioria das questões durante a prática clínica (DYNAMED, 2015). Disponível no portal brasileiro Saúde Baseada em Evidências do Ministério da Saúde (BRASIL, 2015).

UpToDate é um sistema que disponibiliza evidências de apoio à decisão clínica. É escrita por médicos para ajudar médicos a tomar as decisões corretas durante a assistência ao paciente. Seu conteúdo é escrito e editado por uma comunidade global de 5.100 médicos. Possui 46 médicos editores que seguem um processo editorial rigoroso e analisam constantemente o conteúdo informacional produzido a fim de que estejam em conformidade com os últimos achados científicos e clínicos (UPTODATE, 2015).

PUBMED compreende mais de 22 milhões de citações para a literatura biomédica do 
MEDLINE, revistas de ciências da vida, e livros online. Traz a biomedicina e a saúde, abrangendo porções de ciências da vida, ciências do comportamento, ciências químicas e bioengenharia. Também fornece acesso a outros sites relevantes e links para as outras fontes de informação sobre biologia molecular (MEDLINE/PUBMED, 2015).

\section{Método}

O método deste trabalho consistiu em realizar uma revisão da literatura por meio de três etapas distintas: (1) definição das questões de pesquisa; (2) análise bibliométrica; e (3) análise de conteúdo.

Tradicionalmente, uma revisão da literatura pode ser executada por meio de cinco passos: (1) definição das questões de pesquisa; (2) identificação das publicações relevantes; (3) avaliação da qualidade das publicações; (4) síntese das evidências; (5) interpretação dos achados. Com base nestes passos (KHAN; KUNZ; KLEIJNEN, 2003) esta pesquisa adotou:

- Etapa 1 - Definição das questões de pesquisa. As seguintes questões de pesquisa foram consideradas:

(1.1) Como a literatura aborda a temática da utilização das informações de saúde na prática clínica?

(1.2) Qual o significado da informação clínica como instrumento de trabalho dos profissionais de saúde para melhor Prática Baseada em Evidência (PBE)?

- Etapa 2 - Execução de uma análise bibliométrica, com o objetivo de analisar a atividade científica (ou técnica) pelo estudo quantitativo das publicações, o que também permite visualizar de forma integral a bibliografia de determinado campo temático (SENGUPTA, 1992). Para tanto, foram adotados os seguintes passos:

(2.1) Identificação das publicações relevantes;

(2.2) Avaliação da qualidade das publicações;

(2.3) Síntese das evidências.

- Etapa 3 - Análise de conteúdo, com o intuito de realizar a interpretação dos achados decorrentes da pesquisa bibliométrica. Para tanto, foram realizados três passos (baseados em Bardin, 2011):

(3.1) Pré-análise;

(3.2.) Descrição analítica;

\section{POLÊM!CA | Revista Eletronica da Ueji}


(3.3) Interpretação referencial.

Para a seleção do material a ser analisado foi escolhida a base da biblioteca Scientific Electronic Library Online - SciELO (disponível em: http://search.scielo.org/?q=Health\%20Information\%20Systems\%20and\%20Information\%20S ystems\%20and\%20Hospital\%20Information\%20Systems\%20\&where=ORG) e utilizou-se os termos DECS (Descritores em Saúde) que indexam os artigos, combinados com outros termos contidos em títulos e resumos.

A estratégia de busca utilizada foi o uso das seguintes palavras-chave combinadas: Health Information Systems and Information Systems and Hospital Information Systems. A data da coleta foi 24/03/2015 às 00:10h).

A SciELO foi desenvolvida em meados de 2002 pelo projeto de pesquisa da FAPESP Fundação de Amparo à Pesquisa do Estado de São Paulo, em parceria com a BIREME Centro Latino-Americano e do Caribe de Informação em Ciências da Saúde e conta com o apoio do CNPq - Conselho Nacional de Desenvolvimento Científico e Tecnológico. Foi escolhida pois é uma biblioteca eletrônica que abrange uma coleção selecionada de periódicos científicos, especialmente desenvolvidos para responder às necessidades da comunicação nos países em desenvolvimento e particularmente na América Latina e Caribe, assegurando a visibilidade e o acesso universal à literatura científica; tem procedimentos integrados para medir o uso e o impacto dos periódicos, logo, como o presente trabalho buscou entender o significado da informação clínica como instrumento de trabalho dos profissionais de saúde no Brasil, acredita-se que a resposta a essa questão foi devidamente contemplada pelos periódicos indexados nesta base de dados.

Os critérios utilizados para seleção dos artigos consistiram em uma abordagem baseada nos seguintes subtemas, relacionados à informação clínica: esta como dimensão da qualidade do cuidado de saúde; estudos sobre sistemas de informação em saúde. Foram incluídos também na revisão as referências de artigos capturados pela busca eletrônica, livros e documentos de organizações, obtidos em sítios da internet. Foram excluídos artigos cujo objeto de estudo não se referia a questões relacionadas à informação clínica. Foram incluídos artigos sobre o tema da informação clínica no âmbito nacional e internacional. Foram selecionados artigos na Língua Inglesa e Portuguesa, que tivessem disponíveis seus 
respectivos resumos.

A busca eletrônica inicial identificou 115 artigos. Após leitura dos títulos, foram selecionados 52 deles para leitura dos resumos, e finalmente, 12 artigos foram lidos integralmente por um dos autores. A análise do material bibliográfico direcionou a organização do artigo por temas e tomou as definições, as metodologias e os achados descritos nos artigos como aspectos importantes a serem avaliados na revisão.

\section{Resultados}

\section{Qualidade da Informação Clínica}

A informação clínica como instrumento de trabalho dos profissionais de saúde para melhor prática baseada em evidência deve ter qualidade. Existem maneiras de avaliar a qualidade destas informações, por exemplo, os prontuários dos pacientes são analisados por meio de uma comissão de prontuários que se atenta à maneira como as informações são registradas (letra é legível, rasuras, siglas de uso comum ou não, uso de linguagem inapropriada ou registro de conteúdo informacional não relacionado ao paciente; erro na identificação do paciente; registro realizado em prontuário errado; apropriação indevida de informação de paciente; acesso indevido ou não autorizado ao prontuário; analisa o preenchimento de todos os campos dos formulários, se há omissões ou dados incompletos, checagem de medicações, anotações de enfermagem regulares, ausência de identificação do paciente ou do profissional que executou a assistência, dentre outros aspectos), caso haja alguma não conformidade, a mesma tomará as devidas providências (CORREIA, PADILHA, VASCONCELOS, 2014).

Para os artigos científicos indexados nas bases de dados, o principal método de análise da qualidade das informações é o julgamento do método do estudo. Quando dizemos metodologicamente adequados, pretende-se alimentar o reconhecimento das publicações com melhor rigor científico (estudos bem desenhados e bem conduzidos, com cálculo adequado da amostra, compilando esses estudos de forma que possam nortear e reduzir as incertezas clínicas (LOPES, 2002).

As Práticas Baseadas em Evidência não se tratam apenas da utilização de pesquisas publicadas, mas da sistematização e direcionamento das recomendações aplicáveis à prática (GALVÃO, 2012).

A experiência clínica não sistematizada e o racionalismo patofisiológico não podem

\section{POLÊM!CA | Revista Eletronica da Ueji}


dar lugar às tomadas de decisões clínicas, faz-se necessário embasar em pesquisas com boa metodologia, métodos estatísticos adequados, que garantam a qualidade dos resultados finais (CORREIA, PADILHA, VASCONCELOS, 2014).

A associação entre o conhecimento profissional (expertise = interpretação da evidência aplicada no seu paciente) e a evidência científica disponível é a Prática Baseada em Evidência.

Outra questão relacionada à qualidade da informação é o fornecimento do sigilo, das questões éticas além da integração das fontes de informação clínica (os prontuários do paciente) junto com as bases de dados de evidência, respeitando as limitações legais de acesso com foco na melhor decisão assistencial do paciente (LOPES, 2002).

A tecnologia da informação é um instrumento essencial para aproximar e integrar a informação clínica. Existe a informação bibliográfica, que são as bases de evidência conforme descrito neste artigo, em Bases de Dados, e a tecnologia arquivística, que é a informação registrada no prontuário do paciente (MARTINEZ-SILVEIRA, 2003).

Para que a tecnologia da informação seja utilizada de maneira adequada o profissional que irá gerenciá-la deve conhecer os fundamentos da biblioteconomia, da arquivologia, da documentação e da saúde e que esteja disponível para colaborar com a criação e integração de sistemas de informação complexos, assim poderá capacitar os profissionais da saúde a utilizar o sistema de maneira adequada (MARTINEZ-SILVEIRA, 2003).

Segundo Galvão (2012) o caminho da melhor qualidade da informação é integrar um único profissional da informação (seja arquivista, seja bibliotecário) em cada equipe de saúde, da atenção básica em saúde, o que já demandaria cerca de 25.000 profissionais da informação, conhecedores das especificidades da informação clínica. Se fôssemos mais modestos e pensássemos na inserção de um profissional da informação conhecedor da informação clínica em cada município brasileiro, necessitaríamos, ao menos, de 5500 profissionais da informação.

Pelo exposto, é preciso iniciar o trabalho com todos aqueles que estejam ávidos para colaborar com a qualidade da informação clínica, para tomadas de decisão em saúde e para uma assistência em saúde mais resolutiva.

\section{Informação Clínica como Instrumento de Trabalho dos Profissionais de Saúde}

\section{POLÊM!CA | Revista Eletronica da Ueij}


Os profissionais de saúde, o tempo todo, tem a necessidade de obter informações clínicas para melhor assistir o paciente, seja por meio do prontuário com foco na investigação individual ou pelas bases de dados científicas (CORREIA, PADILHA, VASCONCELOS, 2014).

Para que as bases de dados sejam um instrumento de apoio ao profissional é necessário que os serviços de saúde disponibilizem o acesso às mesmas e ofereçam treinamento para capacitar os profissionais a utilizá-las (CORREIA, PADILHA, VASCONCELOS, 2014).

Cabe também relatar que a informação clínica será melhor aproveitada quando há padronização assistencial e evidência dos resultados esperados tanto no diagnóstico como no tratamento do paciente, com isso a redução dos custos assistenciais é notória, além da melhora da qualidade da assistência (MORAES, SANTOS, 2001; CORTES, CORTES, 2011).

Pode-se citar o exemplo da padronização de antibióticos no uso hospitalar, se utilizado um determinado antibiótico para determinada doença por todos os médicos daquela instituição, além da redução do custo por compras devidas, a qualidade da assistência será melhor, pois a prescrição foi baseada em uso prévio, sistematizado e comprovado.

A informação clínica usada de maneira homogênea dentro das instituições de saúde evita conclusões conflitantes. A busca da melhor evidência e das ações baseadas nela com foco no adequado cuidado aos pacientes pelos profissionais de saúde é a escolha mais apropriada para o sucesso assistencial (GALVÃO, 2012).

Identificar e compilar os melhores estudos; avaliar criticamente a literatura disponível; disponibilizar essas evidências em bases de dados eletrônicas são cuidados que o profissional deve ter no uso das informações clínicas (MUELLER, 2000).

A vivência clínica não deve ser descartada, mesmo uma evidência científica de qualidade pode não se aplicar a um determinado paciente.

Estudos clínicos gerados em países ou populações com características diferentes, nem sempre podem ser aplicados ao nosso paciente.

Um protocolo, por exemplo, não pode ser fonte de restrição de uma conduta, a liberdade do profissional conduzir a assistência deve ser respeitada, o que ocorre com o uso da informação clinica é o fato do profissional ter um direcionamento facilitando as suas decisões (BAIN, STANDING, 2009) 
Promover a assistência em saúde com qualidade, estimular a pesquisa clínica e ensino aos profissionais de saúde, auxiliando nas decisões políticas e fornecendo boas práticas com opções baseadas em evidência que permitam decisões apropriadas e racionalização dos recursos é o papel da informação clínica adequada (REIS, MARTINS, LAGUARDIA, 2013).

A difusão da informação em saúde com inúmeros estudos divulgados na internet, congressos e afins, requer uma prática cuidadosa na escolha da informação clínica que o profissional irá embasar-se; verifica-se que transformações importantes ocorreram no cenário moderno e competitivo dos sistemas de cuidados de saúde, sendo a informação clínica essencial neste contexto (GALVÃO, 2012).

Reis, Martins e Laguardia (2013) relatam que no mundo atual a redução da morbimortalidade por diversas doenças contrasta com os riscos elevados inerentes a esses sistemas de informações complexos, com demandas ascendentes e investimentos nem sempre suficientes.

Muitas vezes não existem respostas às perguntas que buscamos, cabendo o desenvolvimento de pesquisas clínicas nestes assuntos.

Cabe lembrar que o foco das informações clínicas é sempre o paciente, sendo necessário uma linguagem clara e objetiva (REIS, MARTINS, LAGUARDIA, 2013).

\section{Conclusão}

Descrever o significado da informação clínica como instrumento de trabalho dos profissionais de saúde para melhor prática baseada em evidência, possibilitou entender que essa ferramenta de trabalho é essencial para uma boa prática clínica, no entanto, faz-se necessário organizar as informações de maneira que sejam eficazes para a tomada de decisão.

A informação clínica é fundamental para os profissionais de saúde, através dela é possível fazer correlações, diagnosticando, tratando e reabilitando adequadamente os pacientes, entretanto, faz-se necessário que se vincule a informação clínica à melhor Prática Baseada em Evidência (PBE), sendo essa relação realizada de maneira sistematizada e integrada, muitas vezes por meio de protocolos e diretrizes.

As tecnologias de informação permitem que os profissionais de saúde individualmente adquiriram software médico ou criem suas bases de dados de forma a armazenar e gerir registros contendo dados relevantes dos seus pacientes, o que ocasiona o não 
compartilhamento com os demais profissionais quando não utilizada, pode ocasionar má qualidade da informação clínica, com não conformidades, inconsistências e/ou redundância nos dados armazenados, além do custo de mão de obra e técnicos, quando não assistenciais, pelo erro na tomada de decisão clínica.

Esta investigação apresenta algumas limitações metodológicas, pois não é um estudo primário, contudo, permitiu a abordagem à problemática em estudo, sob o ponto de vista qualitativo, passível de ser concretizado num estudo posterior de caráter empírico.

\section{Referências}

ASSOCIAÇÃO MEDICA BRASILEIRA. Agência Nacional de Saúde Suplementar (Brasil). Primeira diretrizes clinicas na saúde suplementar - versão preliminar organizado por Agência Nacional de Saúde Suplementa: Rio de janeiro: ANS. 2009

ASSOCIAÇÃO MEDICA BRASILEIRA, CONSELHO FEDERAL DE MEDICINA, AGÊNCIA NACIONAL DE SAÚDE SUPLEMENTAR. O processo da elaboração, validação e implementação das diretrizes clínicas na saúde suplementar no Brasil. Rio de Janeiro: ANS. 2009

BAIN CA, STANDING C. A technology ecosystem perspective on hospital management information systems: lessons from the health literature. Int J Electron Healthc. 2009;5(2):193-210. PubMed PMID: 19906634.

BARDIN, L. Análise de conteúdo. 1a. Ed. São Paulo: Edições 70, 2011.

BESTBETS. Disponível em: http://www.bestbets.org/. Acesso em: 20 de março de 2015.

BRASIL. Ministério da Saúde. Portal da Saúde Baseada em Evidências. Disponível em:

http://portalsaude.saude.gov.br/portalsaude/index.cfm?portal=pagina.visualizarArea\&codArea=392 Acesso em: 23 de março de 2015.

CENTRE for Evidence-Based Medicine. Levels of evidence. Oxford : CEBM, 2011. Disponível em: http://www.cebm.net/index.aspx?o=5653 Acesso em: 20 de março de 2015.

CLINICAL Evidence. Disponível em: http://clinicalevidence.bmj.com/x/index.html Acesso em: 23 de março de 2015.

COCHRANE Systematic Reviews. Disponível em: http://www.cochrane.org/cochrane-reviews. Acesso em: 20 de março de 2015.

CORREIA, L. O. dos S.; PADILHA, B. M.; VASCONCELOS, S. M. L. Métodos para avaliar a completitude dos dados dos sistemas de informação em saúde do Brasil: uma revisão sistemática. Ciênc. saúde coletiva, Rio de Janeiro , v. 19, n. 11, Nov. 2014 Disponível em:

$<$ http://www.scielosp.org/scielo.php?script=sci_arttext\&pid=S1413-81232014001104467\&lng=en\&nrm=iso>. access on 24 Mar. 2015. http://dx.doi.org/10.1590/1413-812320141911.02822013.

CORTES, P. L.; CORTES, E. G. de P. Hospital information systems: a study of electronic patient records. JISTEM J.Inf.Syst. Technol. Manag. (Online), São Paulo, v. 8, n. 1, 2011 Disponível em:

$<$ http://www.scielo.br/scielo.php?script=sci_arttext\&pid=S1807-17752011000100008\&lng=en\&nrm=iso>. Acessado em 2015. http://dx.doi.org/10.1590/S1807-17752011000100008. 
DYNAMED. Disponível em: http://dynamed.ebscohost.com/. Acesso em: 23 de março de 2015.

GALVAO, M.C.B. Informação clínica: do prontuário do paciente às bases de evidência. 23 de agosto de 2012. In: Almeida Junior, O.F. Infohome [Internet]. Londrina: OFAJ, 2012. Disponível em: http://www.ofaj.com.br/colunas_conteudo.php?cod=701 Acessado em: 21/03/2015

GALVAO, M.C.B.; RICARTE, I.L.M. Prontuário do paciente. Rio de Janeiro: Guanabara Koogan, 2012. 344p.

LOPES, I. L. Estratégia de busca na recuperação da informação: revisão da literatura. Ciência da Informação, Brasília, v. 31, n. 2, p. 60-71, maio/ago. 2002.

KHAN, K.S.; KUNZ, R.; KLEIJNEN, J.; ANTES, G. Five steps to conducting a systematic review. Journal of the Royal Society of Medicine, v. 96, p. 118-121, 2003.

MARTINEZ-SILVEIRA, M. S. O bibliotecário e a medicina baseada em evidências. In: ENCONTRO NACIONAL DE CIÊNCIA DA INFORMAÇÃO. 4., 2003, Salvador. Anais. Salvador: UFBA, 2003.

MEDLINE/PUBMED via PICO. Disponível em: http://pubmedhh.nlm.nih.gov/nlmd/pico/piconew.php Acesso em: 23 de março de 2015.

MOTA, L. A. N. da; PEREIRA, F. M. S.; SOUSA, P. A. F. de. Sistemas de Informação de Enfermagem: exploração da informação partilhada com os médicos. Rev. Enf. Ref., Coimbra , v. serIV, n. 1, mar. 2014 . Disponível em <http://www.scielo.mec.pt/scielo.php?script=sci_arttext\&pid=S0874-

02832014000100010\&lng=pt\&nrm=iso>. Acessado em: 25 mar. 2015. http://dx.doi.org/10.12707/RIII12152.

MORAES, I. H. S. de; SANTOS, S. R. F. R. dos. Informações para a gestão do SUS: necessidades e perspectivas. Inf. Epidemiol. Sus, Brasília , v. 10, n. 1, mar. 2001. Disponível em: <http://scielo.iec.pa.gov.br/scielo.php?script=sci_arttext\&pid=S0104-16732001000100006\&lng=pt\&nrm=iso> . Acesso: em 24 mar. 2015. http://dx.doi.org/10.5123/S0104-16732001000100006.

MUELLER, S. P. M. A ciência, o sistema de comunicação científica e a literatura científica. In: CAMPELLO, B. S.; CÉNDON, B. V.; KREMER, J. M. Fontes de Informação para pesquisadores e profissionais. Belo Horizonte: UFMG, 2000. p. 21-34.

OLIVEIRA, S. V. W. B. de et al . Use and development of health information systems: the experience of an organizational unit responsible for the technological services at a public hospital. JISTEM J.Inf.Syst. Technol. Manag. (Online), São Paulo , v. 8, n. 1, 2011 Disponível em: $<$ http://www.scielo.br/scielo.php?script=sci_arttext\&pid=S1807-17752011000100009\&lng=en\&nrm=iso > . Acessado em: 25 Mar. 2015. http://dx.doi.org/10.4301/S1807-17752011000100008.

REIS, C. T.; MARTINS, M.; LAGUARDIA, J. A segurança do paciente como dimensão da qualidade do cuidado de saúde: um olhar sobre a literatura. Ciênc. saúde coletiva, Rio de Janeiro , v. 18, n. 7, July 2013. Disponível em: <http://www.scielo.br/scielo.php?script=sci_arttext\&pid=S1413-

81232013000700018\&lng=en\&nrm=iso>. Acessado em: 24 Mar. 2015. http://dx.doi.org/10.1590/S141381232013000700018.

RODRIGUES, R. J. Information systems: the key to evidencebased health practice. Bulletin of the World Health Organization, Geneve, v. 78, n. 11, p. 1344-1351, 2000.

SACKETT, D. L. et al. Evidence-based medicine: how to practice and teach EBM. Edinburgh: Churchill Livingstone, 1998.

SENGUPTA, I.N. Bibliometrics, informetrics, scientometrics and librametrics: an overview, Libri, n. 42, p. 7598, 1992. 
UPTODATE. Disponível em: http://www.uptodate.com/index. Acesso em: 19 de março de 2015.

Recebido em: 08/04/2015.

Aceito em: 10/06/2015. 